\title{
De UVX 92 (premier laser X saturé) à UVX 2002 (LASERIX), le laser $X$ devient un outil pour les applications scientifiques
}

\author{
G. Jamelot, D. Ros, A. Klisnick, Ph. Zeitoun, J. Dubau, \\ J.-C. Lagron et L. Vanbostal \\ Laboratoire d'Interaction des Rayons X avec la Matière, LIXAM, UMR 8624 du CNRS, \\ Université Paris-Sud, bâtiment 350, 91405 Orsay cedex, France
}

\begin{abstract}
Résumé : Les lasers $X$ pompés par laser ont vigoureusement progressé au cours des 10 dernières années. Les premiers lasers $\mathrm{X}$, quasistationnaires, furent produits dans des installations laser de très grande puissance principalement destinées à la physique de la fusion inertielle. Aujourd'hui, le pompage transitoire nécessite une énergie de pompe beaucoup plus petite et peut être réalisé dans des instaliations laser de taille modeste. LASERIX sera une station de travail destinée spécifiquement à la réalisation de lasers $X$ transitoires, à la recherche de nouveaux schémas de lasers $\mathrm{X}$, ainsi qu'au développement de leurs applications ; le projet est basé sur une chaîne laser de pompe capable de délivrer $10 \mathrm{~J}$ en $500 \mathrm{ps}$ et $10 \mathrm{~J}$ en $1 \mathrm{ps}$.
\end{abstract}

\section{INTRODUCTION}

Les lasers X sont les sources les plus brillantes actuellement réalisées dans le domaine XUV (entre 4 et $50 \mathrm{~nm}$ ). Elles sont produites le plus souvent dans des installations laser de grande puissance destinées principalement aux études de la physique de la fusion inertielle. L'accès très limité à ces installations est actuellement le plus important facteur ralentissant le développement des lasers $\mathrm{X}$ et surtout de leurs applications. La réduction de l'énergie nécessaire au pompage dans le cas des lasers $\mathrm{X}$ transitoires a permis de concevoir des installations laser de puissance moyenne qui sont exclusivement consacrées à ce type de recherche. LASERIX mettra ces sources de rayonnement actuellement inaccessibles à la disposition de la communauté scientifique.

\section{LES LASERS X POMPES PAR LASER}

Les plasmas denses et chauds, et en particulier les plasmas produits par laser ont été considérés dès le début des années 1970 comme le milieu amplificateur de rayons X mous. De nombreux schémas de pompage ont été proposés et étudiés, mais seul le schéma dit collisionnel a jusqu'à présent permis de réaliser des milieux étendus à grand facteur de gain et des lasers $\mathrm{X}$ saturés.

\subsection{Les lasers $X$ collisionnels : le seul schéma de pompage actuellement efficace}

Les lasers $\mathrm{X}$ collisionnels sont des lasers très classiques, à trois niveaux, dont les émetteurs sont des ions multichargés immergés dans un plasma dense. Les niveaux excités (1) et (2) sont peuplés à partir du fondamental (0) par des collisions avec les électrons libres du plasma (fig. 1). Une inversion de population peut être établie entre ces deux niveaux si la force d'oscillateur de la désexcitation radiative $(1) \rightarrow(0)$ est grande, tandis que la transition radiative $(2) \rightarrow(0)$ est strictement interdite. 
Une telle situation se rencontre dans de nombreuses espèces ioniques, par exemple entre les niveaux $2 \mathrm{~s}^{2} 2 \mathrm{p}^{\mathrm{k}-1} 3 \mathrm{p}$ et $2 \mathrm{~s}^{2} 2 \mathrm{p}^{\mathrm{k}-1} 3 \mathrm{~s}$ des ions d'état fondamental $2 \mathrm{~s}^{2} 2 \mathrm{p}^{\mathrm{k}}$ [1]. Ce sont les ions à couches complète, de type néonoïde, nickelloïde ou palladiumoïde qui sont les plus efficaces : ces ions, très stables en température, occupent une zone étendue des plasmas laser, ce qui facilite le transport du rayonnement dans un filament de plasma.

Le problème clé de ce type de laser est de concilier deux exigences contradictoires, d'une part une température de plasma assez basse pour éviter la sur-ionisation et la disparition des ions actifs, d'autre part une population importante d'électrons libres d'énergie suffisante pour exciter les niveaux de la transition laser. Le chauffage rapide du plasma par une impulsion laser permet de satisfaire cette double exigence.

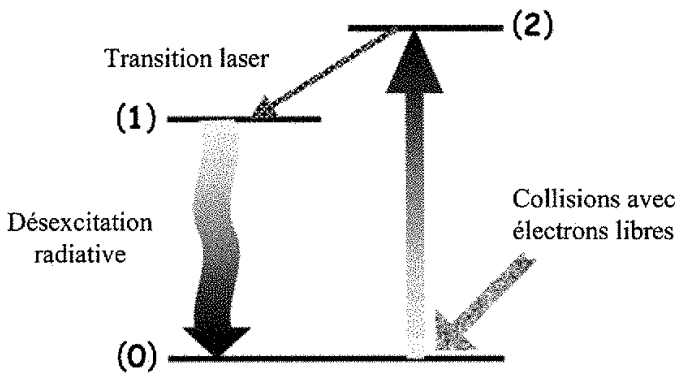

Figure 1 : Schéma de principe d'un laser X collisionnel

\subsection{Les différents types de lasers de pompe}

Le premier laser X a été réalisé en 1984 au Lawrence Livermore National Laboratory (LLNL), pompé par les $4 \mathrm{~kJ}$ du laser "NOVETTE" [2] : deux émission laser étaient observées à 20.6 et $20.9 \mathrm{~nm}$ dans un plasma de sélénium néonoïde. Une meilleure compréhension de l'hydrodynamique du plasma et de la dynamique des populations a abouti en 1993 à la réalisation au LULI d'un laser X saturé à $21.2 \mathrm{~nm}$, avec une énergie de pompe 10 fois plus faible; un gain de $5 \mathrm{~cm}^{-1}$ était mesuré dans le zinc néonoïde [3]. Les inversions "quasistationnaires" (QSS) pompées par des impulsions laser d'environ 500 à 600 ps ont une durée de vie suffisante pour autoriser le double passage du rayonnement dans le filament de plasma, grâce à une demi-cavité constituée d'un miroir interférentiel. Avec un filament de plasma de $2 \mathrm{~cm}$, la demicavité a permis d'atteindre la saturation de l'émission laser.

La réduction de la durée de l'impulsion de pompe dans le domaine de la centaine de picosecondes augmente considérablement le coefficient de gain ; on a ainsi réalisé des lasers X saturés en un seul passage dans le fer néonoïde à $25.5 \mathrm{~nm}$ [4]. Mais l'intérêt majeur des impulsions de 100 ps est qu'elles sont également capables de pomper des inversions dans les ions nickelloïdes, ce qui permet d'atteindre des longueurs d'onde plus courtes, par exemple $13.9 \mathrm{~nm}$ dans le cas de l'argent et même $11.9 \mathrm{~nm}$ avec l'étain. La durée de vie de l'inversion est alors trop courte pour le double passage ; cependant le gain peut être régénéré par un train d'impulsions successives au cours du même tir, ce qui peut permettre des passages multiples dans une cavité résonnante complète et produire un laser $\mathrm{X}$ d'excellente qualité optique.

Les lasers à dérive de fréquence ou "Chirped Pulse Amplification" (CPA), qui délivrent des impulsions ultra courtes allant de quelques femtosecondes à quelques picosecondes, donnent la possibilité de produire des lasers $X$ de très grand gain avec des énergies de pompe réduites. Deux types de pompages collisionnels ont été réalisés avec ces chaines CPA. Dans le cas d'impulsions ultra-courtes et ultraintenses (de l'ordre de $1 \mathrm{~J}$ en $30 \mathrm{fs}$ ) polarisées circulairement et focalisées selon l'axe d'une colonne de gaz, le champ du laser est suffisamment intense pour créer un plasma par effet tunnel. Les électrons libres du plasma sont fortement accélérés par le champ polarisé, et acquièrent une énergie suffisante pour peupler par collision les premiers niveaux excités des ions actifs. Ce schéma de pompage par OFI (Optical Field Ionization) a été mis en œuvre en 1995 [5] et est présenté en détail dans ce volume [6]. 
On peut également utiliser les lasers CPA avec des impulsions un peu plus longues (typiquement 0.5 à 3 ps) en adoptant une technique de focalisation sphérocylindrique semblable à la focalisation utilisée dans le cas quasi-stationnaire. Dans ce cas, il faut produire d'abord un plasma contenant les ions actifs à l'état fondamental, à l'aide d'une impulsion longue de faible énergie. L'absorption de l'impulsion comprimée dans ce plasma chauffe rapidement les électrons libres qui acquièrent une énergie suffisante pour exciter les ions. Il est nécessaire que le front d'énergie de l'impulsion courte soit incliné de $45^{\circ}$ par rapport au plan de la cible (Fig. 2)

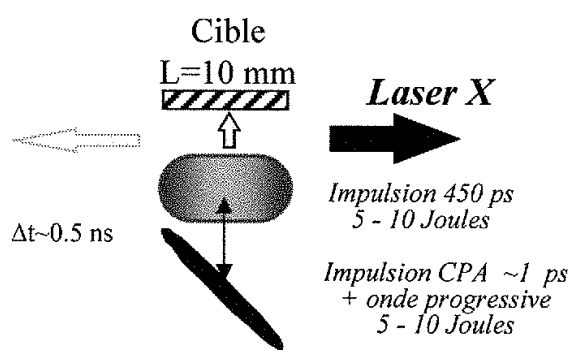

Figure 2 : Principe de fonctionnement d'un laser $\mathrm{X}$ collisionnel transitoire produit à l'aide d'un laser à dérive de fréquence. de façon à ce que l'inversion soit pompée par une onde progressive à la vitesse de la lumière au fur et à mesure de la progression du faisceau laser $X$ dans le plasma [7]. L'énergie nécessaire au pompage transitoire n'est que de l'ordre de $10 \mathrm{~J}$ pour chacune des deux impulsions, ce qui permet de progresser sensiblement sur la voie des "lasers X de table".

\section{APPLICATIONS DES LASERS $X$}

L'intérêt essentiel des lasers $\mathrm{X}$, du point de vue des applications, réside dans leur brillance instantanée exceptionnelle : la courte durée de l'impulsion ( 1 à 80 ps selon le type de pompe utilisée) contenant un nombre élevé de photons $\left(10^{12}\right.$ à $\left.10^{14}\right)$ et la monochromaticité $\left(\lambda / \delta \lambda \cong 10^{4}\right)$ sont des propriétés qui ne se trouvent réunies dans aucune autre source de même longueur d'onde. Divers types d'applications utilisant ces propriétés ont été expérimentés. On peut distinguer les utilisations de lasers $\mathrm{X}$ comme outil de diagnostic (microscopie, interférométrie, holographie à haute résolution, radiographie...) et les utilisations dans lesquelles le laser X est une source d'énergie (luminescence, chauffage de plasma, pompage optique en couche interne, mélange d'ondes, dégradation d'échantillons biologiques...) [8].

La plupart de ces expériences d'application ont été réalisées jusqu'à présent sur des installations laser de grande énergie. L'accès à ces installations est particulièrement limité pour les utilisations ne concernant pas la physique de la fusion inertielle ; de plus leur cadence de tir est très faible, typiquement 3 tirs par heure. De ce fait, chaque expérience n'a disposé que de quelques tirs utiles et n'a fourni dans le meilleur des cas que des résultats très préliminaires. On a pu observer par exemple, in situ, des déformations de surfaces métalliques atteignant quelques nanomètres sous l'effet d'un champ électrique intense, mais il n'a pas été possible de mener une étude systématique impliquant un grand nombre de résultats $[9,10]$. Dans un autre domaine, il n'a pas été possible de réaliser la résolution temporelle de l'émission de bandes UV de luminescence de cristaux ioniques, pourtant nécessaire à l'interprétation correcte de spectres de luminescence excitée par laser X [11].

\section{LASERIX}

L'intérêt des lasers X comme source XUV aux propriétés exceptionnelles ne pourra être démontré que grâce à des installations spécifiques permettant de consacrer un grand nombre de tirs à la réalisation et la caractérisation de lasers $\mathrm{X}$ ainsi qu'à leur utilisation pour des applications variées. De telles installations spécifiques ont été réalisées aux Etats-unis [12] et au Japon [13], d'autres sont en cours d'installation en Allemagne [14]. L'équipe "Lasers X et Applications" du LIXAM a acquis au cours d'expériences au LULI et au Rutherford Appleton Laboratory une maitrise du laser X transitoire; nous avons élaboré à 
partir de ces expériences un projet de station de travail destinée au laser $\mathrm{X}$ transitoire et au développement des applications des lasers X. Ce projet LASERIX a été approuvé par l'université Paris-Sud XI, qui l'a incorporé au projet PÔLA, financé dans le cadre du CPER 2000-2006.

\subsection{Les choix technologiques de la chaîne laser de pompe}

La chaîne laser de pompe constitue la partie la plus importante du projet, qui comprend en outre une enceinte de production de plasma amplificateur et deux enceintes pour l'accueil des expériences d'application.

Les installations "lasers $X "$ actuelles sont toutes basées sur des chaînes de pompe CPA dont la partie "amplification à granđe énergie" est formée de barreaux de verre dopé au néodyme, le "front end" utilisant la technologie "titane:saphir". Les progrès techniques concernant la croissance des cristaux de Ti:Sa ont permis récemment de porter à $15 \mathrm{~J}$ l'énergie d'un laser Ti:saphir [15]; cette valeur pourrait être augmentée notablement. La cadence de tir de la chaîne de pompe pourrait être augmentée de près de deux ordres de grandeur pour atteindre $0.1 \mathrm{~Hz}$ en remplaçant le Nd:verre par le Ti:saphir, ce qui rendrait l'installation très compétitive. Nous étudions actuellement en collaboration avec le LOA les possibilités de réaliser ce saut technologique dans le cadre du projet LASERIX.

\section{CONCLUSION}

LASERIX sera à l'échéance de 2005 une installation destinée aux progrès des sources "laser X", mais ce sera aussi et surtout une installation ouverte à l'ensemble de la communauté scientifique pour développer des applications nécessitant de très grandes brillances de source.

\section{Remerciements}

LASERIX est étudiée actuellement avec l'aide de nombreux collègues de divers laboratoires français : CEA, CELIA, GREMI, LCFIO, LIXAM, LOA, LPGP, etc. Nous les remercions de leur soutien, gage de l'efficacité de la future installation. La naissance de ce projet doit beaucoup à Pierre Jaeglé, qui a joué un rôle primordial dans la réussite des recherches sur le laser $\mathrm{X}$ en France.

\section{Références}

1 Elton R.C., Applied Optics, 14 (1975) 97-101.

2 Matthews D.L. et al, Phys. Rev. Letters, 54 (1985) 110-3 http:/www.mbi-berlin.de/en/research/projects/I.6/i6e.htm

3 Rus B. et al., J. Opt. Soc. Am. B, 11 (1994) 564.

4 Sebban S. et al, Proceedings of the SPIE 3156 (1997) 11-20

5 Lemoff B.E. et al., Phys. Rev. Lett. 74 (1995)1574.Ò

6 Sebban S. et al, dans ce volume

7 Klisnick, A. et al., J. Opt. Soc. Am. B 17 (2000) 1093-7

8 voir "X-Ray Lasers 2000", édité par G. Jamelot, C. Möller, et A. Klisnick, J. Phys. IV France 11 (2001) Pr2-

9 Albert F. et al., Phys. Rev. B, 60 (1999)11089-94

10 Mocek T. et al., dans ce volume

11 Belsky A.N., et al., J. Phys. IV France 11 (2001) Pr2-495

12 Dunn J. et al., X-ray Lasers 1998, Inst. Phys. Conf. Ser. N 159, IOP Publishing, Bristol (1999) 51-8

13 Kato Y. et al., J. Phys. IV France 11 (2001) Pr2 - 3-10

14 http://www-aix.gsi.de/ phelix/, http://www.mbi-berlin.de/en/research/projects/L.6/i6e.htm

15 Bonlie J.D. et al., Appl.Phys. B, 70 (2000) S155-60 